\title{
Advances in cancer biology and therapy
}

\author{
Gregg L. Semenza
}

Published online: 21 March 2013

(C) Springer-Verlag Berlin Heidelberg 2013

This issue of the Journal of Molecular Medicine contains three outstanding review articles that summarize recent advances in cancer metastasis and anti-angiogenic therapy by leaders in the field. Interactions between cancer cells and stromal cells play critical roles in the metastatic process, which in turn is the major cause of cancer mortality. Among the stromal cell types that are discussed in these review articles are cancer-associated fibroblasts, inflammatory cells, and endothelial cells.

Smith and Kang review the rapidly growing body of data regarding the mechanisms by which many different inflammatory cell types, including macrophages, myeloid-derived suppressor cells, neutrophils, regulatory $\mathrm{T}$ cells, and suppressive dendritic cells, are recruited to primary tumors and promote metastasis [1]. In addition, factors secreted by primary tumors also recruit bone marrow-derived cells to future sites of metastasis in the lungs and other organs, as part of the process known as pre-metastatic niche formation.

Kahlert and Kalluri review the role of exosomes in cancer progression and metastasis [2]. Exosomes are membrane vesicle nanoparticles containing proteins, mRNAs, and microRNAs that are released by cancer cells and change the biological properties of stromal cells to support cancer progression. The potential use of exosomes as biomarkers in cancer diagnosis and therapy is also discussed.

One of the unfulfilled promises of cancer research over the last decade has been the targeting of tumor angiogenesis for cancer therapy. Verma and colleagues review the multiple mechanisms by which glioblastoma multiforme, which is a highly vascularized and invasive brain cancer, becomes resistant to anti-angiogenic therapy [3]. They also discuss the mechanisms by which treatment with angiogenesis inhibitors may promote invasive properties of cancer cells.

Taken together, these three reviews highlight the increasing complexity of cancer biology. One consequence of this complexity is an appreciation that successful therapy will require combinations of drugs that reduce the probability of resistance or counter-therapeutic effects and that target both cancer and stromal cell populations.

\section{References}

1. Smith HA, Kang Y (2013) The metastasis-promoting roles of tumor-associated immune cells. J Mol Med. doi:10.1007/s00109013-1021-5

2. Kahlert C, Kalluri R (2013) Exosomes in tumor microenvironment influence cancer progression and metastasis. J Mol Med. doi:10.1007/s00109-013-1020-6

3. Soda Y, Yskiw C, Rommel A, Verma IM (2013) Mechanisms of neovascularization and resistance to anti-angiogenic therapies in glioblastoma multiforme. J Mol Med. doi:10.1007/s00109-0131019-z
G. L. Semenza $(\bowtie)$

Vascular Program, Institute for Cell Engineering; Departments of Pediatrics, Medicine, Oncology, Radiation Oncology, and Biological Chemistry; and McKusick-Nathans Institute of Genetic Medicine, Johns Hopkins University School of Medicine,

Baltimore, MD 21205, USA

e-mail: gsemenza@jhmi.edu 